Int. J. Odontostomat., 8(3):439-445, 2014.

\title{
Expresión de E-Cadherina y Syndecan-1 en Liquen Plano de Piel y de Mucosa Oral
}

\author{
Expression of E-Cadherin and Syndecan-1 in Oral and Skin Lichen Planus
}

\author{
Carreon-Burciaga, R. G.; Mendoza-Roaf, P.*; Amezcua-Rosas, G. ${ }^{* * *}$; Bologna-Molina, R. ; \\ Gonzélez-Montemayor, T.*; González-González, R." \& Lemús-Rojero, O. ${ }^{* * *}$
}

CARREON-BURCIAGA, R. G.; MENDOZA-ROAF, P.; AMEZCUA-ROSAS, G.; BOLOGNA-MOLINA, R.; GONZÉLEZMONTEMAYOR, T.; GONZÁLEZ-GONZÁLEZ, R. \& LEMÚS-ROJERO, O. Expresión de E-Cadherina y Syndecan-1 en liquen plano de piel y de mucosa oral. Int. J. Odontostomat., 8(3):439-445, 2014.

RESUMEN: El liquen plano es una lesión inmunológica que se caracteriza por presentar apoptosis de los queratinocitos basales, licuefacción de la membrana basal e infiltrado linfoplasmocitario en banda, estas características pudieran estar asociadas con la pérdida de adhesiones celulares y riesgo de transformación maligna. Para identificar el potencial regulatorio asociado a la adhesión celular de los queratinocitos basales y la posible capacidad de transformación maligna, se investigó la expresión "in situ" de E-cadherina y Syndecan-1 el liquen plano de piel y de mucosa oral. En un total de 37 casos de liquen plano de piel y 5 casos de liquen plano oral, se realizaron estudios de inmunohistoquímica para la detección de Ecadherina y Syndecan-1. En las áreas de enfermedad activa del liquen plano de piel los queratinocitos basales no expresaron E-cadherina y la expresión de Syndecan-1 fue focal, en las áreas de tejido epitelial libre de enfermedad la expresión de ambas proteínas fue muy similar. Los cambios de expresión de las proteínas E-cadherina y Syndecan-1 sugieren una posible asociación de las mismas a la patogénesis de Liquen plano de piel y de mucosa oral.

PALABRAS CLAVE: liquen plano, E- Cadherin, Syndecan-1, transformación maligna.

\section{INTRODUCCIÓN}

El liquen plano (LP) es una condición inflamatoria que afecta a los epitelios estratificados, se presenta aproximadamente en el $1-2 \%$ de la población y se observa con mayor frecuencia en mujeres (Axéll \& Rundquist, 1987), aunque su etiología es desconocida, es posible que intervengan diversos factores que induzcan la activación de linfocitos en contra de los queratinocitos basales (Ebrahimi et al., 2008; Neppelberg et al., 2007; Zyada \& Fikry, 2010) induciendo de esta forma muerte celular por apoptosis (Santoro et al., 2004; Sugerman et al., 2000).

Los queratinocitos tienen la capacidad de poder expresar diversas moléculas de adhesión que tienen funciones importantes para las uniones inter-celulares y con matriz extra celular (Presland \& Jurevic, 2002;
Gooding et al., 2004) E-cadherina es una proteína transmembranal de adhesión cuya principal función es participar en las adhesiones célula-célula del epitelio escamoso estratificado, se expresa en mucosa oral y piel y mantiene los contactos celulares que pueden prevenir la muerte celular por apoptosis en líneas celulares inmortalizadas (Galaz et al., 2005). La pérdida de expresión de E-cadherina está relacionada con diversos tumores malignos y dicha falta de expresión puede asociarse con pérdida de adhesión celular, lo que pudiera estar relacionado con invasión y avance tumoral (Mohtasham et al., 2014). Syndecan es una familia de proteoglucanos de superficie celular que interactua con varios efectores moleculares de la matriz extracelular y factores de crecimiento (Bernfield \& Sanderson, 1990; Inki et al., 1994). Syndecan-1 es el miembro de la fa-

\footnotetext{
Facultad de Odontología, Universidad Juárez del Estado de Durango, Durango, México.

* Departamento de Disciplinas Filosófico, Metodológicas e Instrumentales, Centro Universitario de Ciencias de la Salud, Universidad de Guadalajara, Guadalajara, México.

*** Instituto Dermatológico de Jalisco "Dr. José Barba Rubio", Jalisco, México.

${ }^{* * * *}$ Facultad de Odontología, Universidad Autónoma de Zacatecas, Zacatecas, México.

Este trabajo fue financiado por el Programa de Mejora del Profesorado (PROMEP) Secretaría de Educación Pública (SEP) México.
} 
CARREON-BURCIAGA, R. G.; MENDOZA-ROAF, P.; AMEZCUA-ROSAS, G.; BOLOGNA-MOLINA, R.; GONZÉLEZ-MONTEMAYOR, T.; GONZÁLEZ-GONZÁLEZ, R. \& LEMÚSROJERO, O. Expresión de E-Cadherina y Syndecan-1 en liquen plano de piel y de mucosa oral. Int. J. Odontostomat., 8(3):439-445, 2014.

milia que se ha estudiado de manera más extensa, se localiza principalmente en células epiteliales y su expresión esta relacionada al desarrollo embrionario (Zyada \& Fikry), Syndecan-1 participa mediando la adhesión celular con diversas moléculas de la matriz extracelular y actúa como co-receptor para factores de crecimiento fibroblástico, también es un potente factor de crecimiento angiogénico involucrado en la diferenciación (Stanley et al., 1995; Inki \& Jalkanen, 1996) La sobreexpresión, disminución o pérdida de la expresiónde Syndecan-1 esta relacionado con diversos tumores malignos (Inki \& Jalkanen). La sobreexpresión, disminución o la completa ausencia de expresión de Syndecan-1 ha sido estudiada en diversos tipos de carcinomas asociándose al comportamiento clínico (Anttonen et al., 1999; Matsumoto et al., 1997; Bologna-Molina et al., 2008; Saunders et al., 1989).

Mientras que el LP de la piel es autolimitado, las lesiones bucales son crónicas y potencialmente premalignas. La importancia del diagnóstico y control del LP en cavidad bucal radica en que es una enfermedad considerada como cancerizable. Se han realizado diversos estudios para determinar la frecuencia de la transformación maligna del LP cuando se manifiesta en cavidad bucal, dicha transformación varía desde un 0,3 a un $10 \%$, con una media reportada del 2\% (Axéll \& Rundquist; Ebrahimi et al.; Neppelberg et al.; Zyada \& Fikry).

El propósito de este estudio fue evaluar la expresión de E-cadherina y Syndecan-1 en Liquen plano oral (LPO) y Liquen plano de piel (LPP) para establecer una posible asociación de la expresión de estas proteínas con transformación maligna.

\section{MATERIAL Y MÉTODO}

Fueron estudiados un total de 42 casos de LP, las muestras fueron obtenidas del Instituto Dermatológico de Jalisco “Dr. José Barba Rubio”, diagnosticadas durante el periodo comprendido del 2004 al 2012 y que correspondieron a LP con manifestaciones exclusivas de piel (LPP) y casos de LP con manifestación exclusiva en cavidad bucal (LPO). Los especímenes estaban conservados en bloques de parafina. Se realizaron cortes de los tejidos y estos fueron teñidos con hematoxilina y eosina. El estudio histopatológico se realizó por dos patólogos con experiencia en LP tomando en cuenta los parámetros publicados por Fernández-González et al. (2011).
Inmunohistoquímica. Los estudios de inmunohistoquímica se realizaronen la Facultad de Odontología de la Universidad Juárez del Estado de Durango, se hicieron cortes de $2 \mathrm{~mm}$ de espesor, los cuales se montaron sobre laminillas tratadas con poly-L-lisina, posteriormente los cortes fueron desparafinados en una estufa a $60^{\circ} \mathrm{C}$ por 30 minutos y se colocaron en xilol por 5 minutos. Los cortes se hidrataron en un tren de concentraciones decrecientes de alcoholes (absoluto, 90, 70 y 50\%) y enjuagues con agua destilada. Para el desenmascaramiento de los epítopes se realizó recuperación antigénica con solución de citrato de sodio $10 \mathrm{mM}$, con pH bajo para Syndecan-1 y alto para E-cadherina, dicha recuperación se hace en una olla de presión para microondas con potencia máxima de 750 w. Las peroxidasas endógenas fueron bloqueadas con peróxido de hidrógeno al 0,9\%, seguidas por lavados con agua destilada y solución salina amortiguada de fosfatos $\mathrm{pH}$ 7,4 (PBS). Los anticuerpos primarios se incubaron por un tiempo de 30 minutos contra: E-cadherina dilución 1:100 (Clone NCH-38, Monoclonal Mouse, Anti-Human, DakoCorp) y Syndecan-1 dilución 1:100 (CD-138, Clone MIB 15, Monoclonal Mouse, Anti - Human, DakoCorp). Posteriormente, los cortes se incubaron con el segundo anticuerpo biotinilado anti ratón/anti conejo y con el complejo streptavidina/peroxidasa (LSA-B+Dako Corporation, Carpintería CA, USA) por 30 minutos cada uno. Los productos de la reacción se visualizaron con sustrato de 3,3'-diaminobenzidina$\mathrm{H}_{2} \mathrm{O}_{2}$ (Dako Corporation, Carpintería, CA, USA). Como controles positivos se utilizaron fragmentos de mucosa oral y piel y para los controles negativos se omitió la incubación con los anticuerpos primarios.

El porcentaje de E-cadherina y Syndecan-1 de las células positivas fue calculado usando un microscopio Nikon Eclipse E200 con objetivo de 10x, correspondiendo a un área de $5,3 \mathrm{~mm}^{2}$. Con la ayuda de una cámara digital (Olympus C-7070) se tomaron microfotografías de cinco campos seleccionados al azar en donde se observaron mayor área de daño epitelial por respuesta inflamatoria.

El análisis estadístico fue realizado usando la $\mathrm{X}^{2}$ de Pearson para comparar proporciones y ANOVA para comparar las medias de expresión inter e intragrupos, y observar las posibles diferencias entre LPP y LPO. Los resultados fueron considerados significantes para un $p<0,05$. Para el análisis estadístico se utilizó el programa SPSS 15.0 (SPSS Profesional Statistics, SPSS Inc., Chicago IL). 
CARREON-BURCIAGA, R. G.; MENDOZA-ROAF, P.; AMEZCUA-ROSAS, G.; BOLOGNA-MOLINA, R.; GONZÉLEZ-MONTEMAYOR, T.; GONZÁLEZ-GONZÁLEZ, R. \& LEMÚSROJERO, O. Expresión de E-Cadherina y Syndecan-1 en liquen plano de piel y de mucosa oral. Int. J. Odontostomat., 8(3):439-445, 2014.

\section{RESULTADOS}

Treinta y siete casos $(88,1 \%)$ de 42 correspondieron exclusivamente a LPP y cinco $(11,9 \%)$ correspondieron exclusivamente a LPO. EI LPP se presentó en una relación de Hombre:Mujer de 1:1,1 y en LPO la relación fue de 4:1. El promedio de edad fue bimodal cuyos picos de edad fueron de los 30 a 35 años y de $>65$ años. La edad mínima de presentación fue de 10 años y la máxima de 71 años de edad (Tabla I).

Tabla I. Distribución por edad y sexo del liquen plano Los valores en negritas indican el predominio de distribución de Liquen plano, en relación con el sexo y sitio de localización.

\begin{tabular}{|c|c|c|c|}
\hline \multirow[b]{3}{*}{ Localización } & \multicolumn{2}{|c|}{ Sexo } & \multirow{3}{*}{$\begin{array}{l}\text { Total } \\
\text { n (\%) }\end{array}$} \\
\hline & Masculino & Femenino & \\
\hline & n (\%) & n (\%) & \\
\hline Piel & $17(54)$ & $20(46)$ & $37(100)$ \\
\hline Mucosa oral & $4(90)$ & $1(10)$ & $5(100)$ \\
\hline Total & $21(50)$ & $21(50)$ & $42(100)$ \\
\hline
\end{tabular}

E-cadherina inmunoreactividad. La expresión de Ecadherina fue considerada positiva cuando la inmunoreactividad fue inequívocamente observada en la membrana y/o citoplasma celular. La inmunopositividad para E-cadherina se observó en el epitelio estratificado tanto de la mucosa oral y piel.

Expresión de E-cadherina en queratinocitos basales en áreas con daño epitelial de LPP. En el LPP el porcentaje de expresión predominante de E- cadherina fue de $<50 \%$ con un total de 15 casos, dicha expresión se observó principalmente en las zonas que expresaron inflamación subepitelial o destrucción de células basales. La expresión $>50 \%$ de ECadherina se observó en cuatro casos y dicha expresión estaba presente en zonas donde la inflamación subepitelial y/o destrucción de las células basales no era tan evidente (Fig. 1a).

\section{Expresión de E-cadherina en queratinocitos} basales en áreas con daño epitelial de LPO. En el LPO la expresión fue de $<50 \%$ en tres casos y al igual que en el LPP dicha expresión estuvo asociada a zonas donde la inflamación subepitelial y/o destrucción de células basales era predominante (Fig. 1b).

Syndecan-1 inmunoreactividad. Al igual que Ecadherina, la expresión de Syndecan-1 fue considerada positiva cuando la inmunoreactividad fue inequívocamente observada en la membrana o citoplasma celular. Syndecan-1 se observó en los epitelios estratificados de mucosa y piel normales, en algunos casos se llegó a observar una línea de color café confinado a la membrana basal. Diferentes porcentajes de inmunopositividad se observaron en las lesiones de LP y al igual que E-cadherina en los casos de LPP en las zonas que expresaron inflamación subepitelial y/o destrucción de células basales solo algunos queratinocitos (26-50\%) expresaron Syndecan-1, mientras que LPO, la expresión de Syndecan-1 fue del $100 \%$ (Fig. $2 a$ y $2 b$ ).

E-cadherina y Syndecan-1 inmunoreactividad. La disminución de la expresión de E-cadherina en las

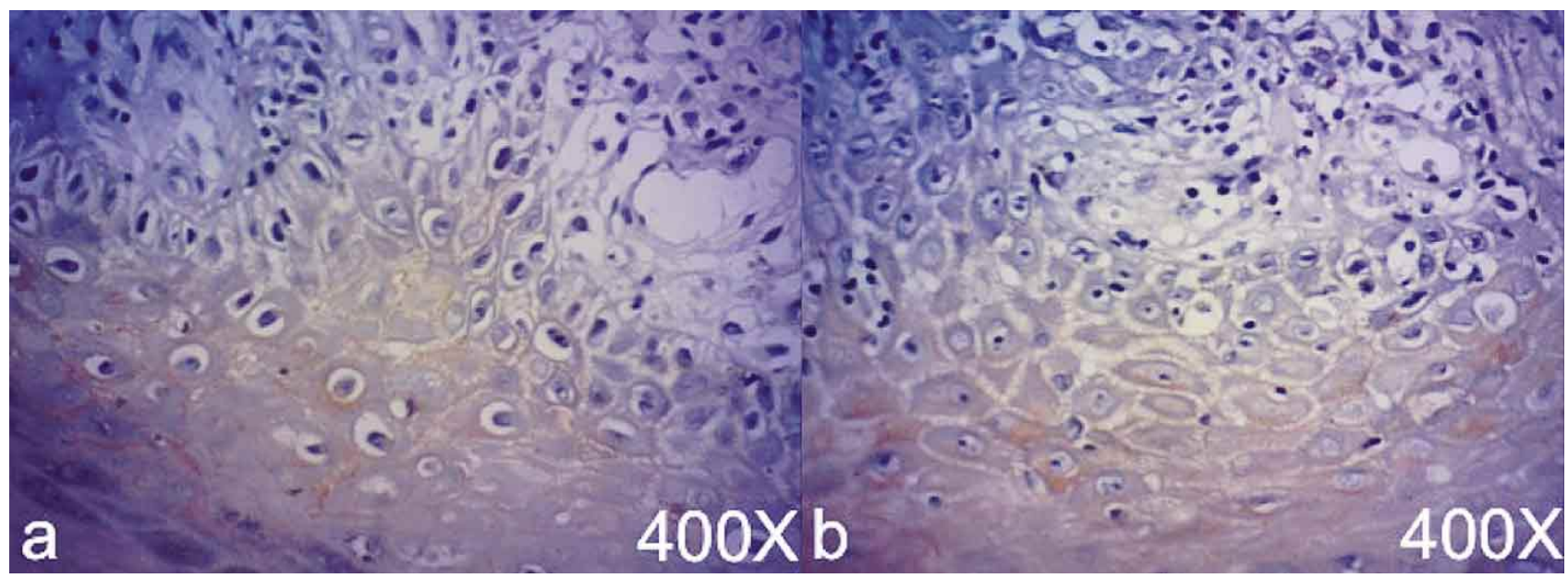

Fig. 1. Expresión de E-cadherina en a) Liquen plano en piel (LPP) y b) Liquen plano oral (LPO). Obsérvese la disminución de la expresión en áreas dónde esta presente la respuesta inflamatoria, licuefacción de la membrana basal y filtración de linfocitos al epitelio escamoso. 


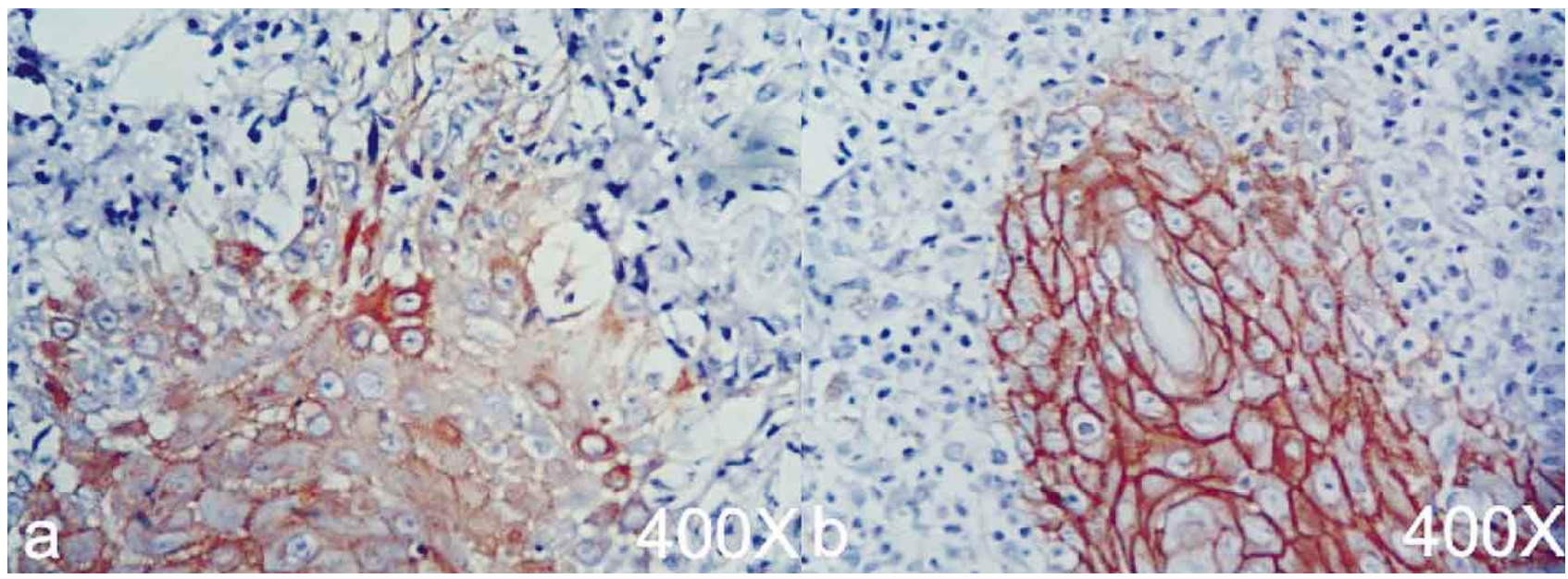

Fig. 2. Expresión de Syndecan-1 en a) Liquen plano en piel (LPP) y b) Liquen plano oral (LPO). Obsérvese la disminución de la expresión de Syndecan-1 en LPP, mientras que en LPO no se observa una disminución en la expresión de Syndecan-1.

Tabla II. Relación entre la expresión de E-cadherina y Syndecan-1 en Liquen plano de piel y Liquen plano oral.

\begin{tabular}{llccc}
\hline & Porcentaje de Expresión & LPP n, (\%) & LPO n, (\%) & $\boldsymbol{p}=$ \\
\hline E-cadherina & $\mathbf{0}$ & $13(40,6)$ & $2(40)$ & \\
& $\mathbf{1 - 2 5 \%}$ & $7(21,9)$ & $1(20)$ & \\
& $\mathbf{2 6 - 5 0 \%}$ & $8(25)$ & $2(40)$ & 0,747 \\
& $\mathbf{5 1 - 7 5 \%}$ & $2(6,3)$ & 0 & \\
Syndecan-1 & $\mathbf{7 6 - 1 0 0 \%}$ & $2(6,2)$ & 0 & \\
& $\mathbf{0}$ & 0 & $0(0)$ & \\
& $\mathbf{1 - 2 5 \%}$ & 0 & $0(0)$ & 0,583 \\
& $\mathbf{2 6 - 5 0 \%}$ & $1(2,7)$ & $0(0)$ & \\
& $\mathbf{5 1 - 7 5 \%}$ & $2(5,4)$ & $0(0)$ & \\
& $\mathbf{7 6 - 1 0 0 \%}$ & $34(91,8)$ & $5(100)$ & \\
\hline
\end{tabular}

La expresión predominante de E-cadherina en Liquen plano de piel (LPP) y Liquen plano oral (LPO), fue del $50 \%$ o menos, mientras que la expresión de Syndecan-1 fue mayor al $50 \%$.

zonas de inflamación subepitelial o destrucción de células basales fue en mayor porcentaje que la expresión por Syndecan-1, es decir siete casos de LPP y un caso de LPO presentaron disminución de expresión de E-cadherina (1-25\%), mientras que Syndecan1 se vio conservada en casi la totalidad de los casos (Tabla II).

\section{DISCUSIÓN}

En el presente estudio la expresión de ECadherina en el LPP y LPO fue significativamente menor en comparación con la expresión de Syndecan1. Este resultado puede estar relacionado a que la disminución de la proteína E-cadherina está estrechamente relacionada con la pérdida en la adhesión celular, que es fundamental para mantener la arquitectura y homeostasis de las células epiteliales y es nece- saria para la migración y la transducción de señales intracelulares (Galaz et al.; Doddawad, 2014) EI LP es una enfermedad autoinmune en la que participan principalmente linfocitos T CD4 y CD8, la infiltración de los linfocitos activa las vías de apoptosis en los queratinocitos del estrato basal, dicha infiltración ocasiona un incremento de apoptosis en los queratinocitos y una disminución de apoptosis en los linfocitos T (Lei et al., 2010; Hadzi-Mihailovic et al., 2009). Este mecanismo inmunológico autodestructivo es más abundante en el LPO que en LPP (Santoro et al.) y es provocado por una respuesta inmune alterada que resulta de apoptosis basilar de los queratinocitos y encabeza la licuefacción de la capa basal del epitelio (Gorouhi et al., 2014), lo que ocasiona pérdida de adhesiones celulares y disminución de la expresión de E-cadherina en los estratos basales. Neppelberg et al. reportan que la pérdida de la expresión de E-cadherina esta relacionada a la patogénesis del LPO. La pérdida de Ecadherina en los queratinocitos basales puede contri- 
CARREON-BURCIAGA, R. G.; MENDOZA-ROAF, P.; AMEZCUA-ROSAS, G.; BOLOGNA-MOLINA, R.; GONZÉLEZ-MONTEMAYOR, T.; GONZÁLEZ-GONZÁLEZ, R. \& LEMÚSROJERO, O. Expresión de E-Cadherina y Syndecan-1 en liquen plano de piel y de mucosa oral. Int. J. Odontostomat., 8(3):439-445, 2014.

buir a la reducción de la integridad estructural de las células basales lo que pudiera permitir la migración de las células $T$ a los compartimientos epiteliales en el LPO (Neppelberg et al.). Aunque existen pocos estudios que relacionan la expresión de E-cadherina con el LPP, en nuestro trabajo se observó una evidente pérdida en la expresión de E-cadherina, estos datos pueden sugerir que el LPP presenta características semejantes al LPO y que la pérdida de la expresión de E-cadherina posiblemente esté relacionada también con la patogénesis del LPP.

La reducción en la expresión de Syndecan-1 está presente durante la transformación maligna de varios epitelios y la pérdida se correlaciona con los grados de diferenciación histológica de los carcinomas escamosos y la falta de expresión está asociada a tumores pobremente diferenciados. En los carcinomas de cabeza y cuello la expresión positiva de syndecan-1 se correlaciona con un pronóstico favorable (Inki \& Jalkanen). Estudios experimentales indican que la expresión de Syndecan-1 esta asociada con el mantenimiento de la morfología epitelial, el crecimiento dependiente del anclaje celular y la inhibición de invasión in vitro (Reiland et al., 2004; Tsanou et al., 2004; Juuti et al., 2005) Estudios realizados por Zyada \& Fikry indican que la intensidad en la expresión de Syndecan-1 esta relacionada con las variantes clínicas de LPO siendo la variante reticular la que presenta una mayor expresión de Syndecan-1. En nuestro estudio únicamente se incluyó la variante reticular de LPO y se observó que el porcentaje de expresión de syndecan-1 fue $>75 \%$ en LPO y LPP. Zyada \& Fikry indican que las lesiones de LPO que expresan una fuerte inmunoexpresión de Syndecan-1 no progresan a carcinoma, en su estudio encontraron que tres lesiones de LPO que progresaron a carcinoma de células escamosas evidenciaban una baja regulación de la expresión de Syndecan-1. Tomando en cuenta estos estudios previos y lo encontrado en nuestro trabajo podemos sugerir que la expresión de Syndecan-1 en el LP pude ser un marcador importante para evaluar el potencial de transformación maligna de lesiones de LP. Souka et al. (2000) encontraron una elevada expresión de Syndecan-1 en células de epitelio normal, mientras que en los epitelios displásicos y en las islas tumorales de carcinomas invasores la expresión de Syndecan-1 estaba disminuida o completamente ausente. Por lo cual pudiéramos interpretar que la pérdida de la expresión de Syndecan-1 pudiera estar asociada con proliferación no controlada, reducción de adhesión celular y alteración en la diferenciación celular en el LP (Inki \& Jalkanen; Souka et al.).

En conclusión, los resultados del presente estudio dan información relevante nos hace proponer que la disminución o la pérdida de expresión de E-cadherina en los queratinocitos basales está relacionado con la patogénesis del LP, en particular afectando la integridad del epitelio basal y con la consiguiente capacidad de migración de linfocitos T en el epitelio del LP.

Basándonos en nuestros resultados y en lo reportado en la literatura en lesiones epiteliales premalignas y malignas podemos sugerir que la expresión de Syndecan-1 pudiera estar asociada al riesgo de transformación maligna en el LPO. Cabe hacer notar que nuestro estudio presenta limitantes debido al número de casos estudiados, en especial en los casos de LPO; por tanto el afirmar que la ausencia o disminución de la expresión de Syndecan-1 esta relacionado con la transformación maligna no es posible aún, se requiere realizar estudios con más casos, en los cuales además de estudiar la expresión de esta proteína directamente en el tejido, se relacionen factores endógenos y exógenos de riesgo que pudieran intervenir en la transformación maligna de esta discutida lesión.

CARREON-BURCIAGA, R. G.; MENDOZA-ROAF, P.; AMEZCUA-ROSAS, G.; BOLOGNA-MOLINA, R.; GONZÉLEZMONTEMAYOR, T.; GONZÁLEZ-GONZÁLEZ, R. \& LEMÚS-ROJERO, O. Expression of E-Cadherin and Syndecan-1 in oral and skin lichen planus. Int. J. Odontostomat., 8(3):439-445, 2014.

SUMMARY: Lichen planus is an immunological lesion is characterized by basal keratinocytes apoptosis, liquefaction of the basal membrane and linphoplasmocitary infiltration, these features may be associated with loss of cellular adhesion and risk of malignant transformation. To identify the regulatory potential associated with cell adhesion of basal keratinocytes and the malignant transformation potential, the "in situ" expression of E-cadherin and Syndecan-1 proteins in skin and oral lichen planus were investigated. A total of 37 cases of skin lichen planus and 5 cases of oral lichen planus we reevaluated by immunohistochemical approach, using E-cadherin and Syndecan-1antibodies. In areas of active disease in skin lichen planus the basal keratinocytes did not express E-cadherin and Syndecan-1 expression was focal, in the of epithelial tissue areas of free lesion the expression of both proteins were similar. Changes in E-cadherin and Syndecan-1 expression suggest a possible association of this proteins with the pathogenesis of skin and oral lichen planus.

KEY WORDS: lichen planus, E-Cadherin, Syndecan-1, malignant transformation. 


\section{REFERENCIAS BIBLIOGRÁFICAS}

Anttonen, A.; Kajanti, M.; Heikkilä, P.; Jalkanen, M. \& Joensuu, $\mathrm{H}$. Syndecan-1 expression has prognostic significance in head and neck carcinoma. Br. J. Cancer, 79(3-4):558-64, 1999.

Axéll, T. \& Rundquist, L. Oral lichen planus--a demographic study. Community Dent. Oral Epidemiol., 15(1):52-6, 1987.

Bernfield, M. \& Sanderson, R. D. Syndecan, a developmentally regulated cell surface proteoglycan that binds extracellular matrix and growth factors. Philos. Trans. R. Soc. Lond. B. Biol. Sci., 327(1239):171-86, 1990.

Bologna-Molina, R.; Mosqueda-Taylor, A.; LopezCorella, E.; Almeida, O. P.; Carrasco-Daza, D.; Garcia-Vazquez, F.; Farfan-Morales, J. E.; IrigoyenCamacho, M. E. \& Damián-Matsumura, P. Syndecan-1 (CD138) and Ki-67 expression in different subtypes of ameloblastomas. Oral Oncol., 44(8):805-11, 2008.

Doddawad, V. G. Histopathological analysis of apoptotic cell count and its role in oral lichen planus. J. Oral Maxillofac. Pathol., 18(1):42-5, 2014.

Ebrahimi, M.; Boldrup, L.; Wahlin, Y. B.; Coates, P. J. \& Nylander, K. Decreased expression of the p63 related proteins beta-catenin, E-cadherin and EGFR in oral lichen planus. Oral Oncol., 44(7):634-8, 2008.

Fernández-González, F.; Vázquez-Álvarez, R.; Reboiras-López, D.; Gándara-Vila, P.; GarciaGarcia, A. \& Gándara-Rey, J. M. Histopathological findings in oral lichen planus and their correlation with the clinical manifestations. Med. Oral Patol. Oral Cir. Bucal, 16(5):e641-6, 2011.

Galaz, S.; Espada, J.; Stockert, J. C.; Pacheco, M.; Sanz-Rodríguez, F.; Arranz, R.; Rello, S.; Cañete, M.; Villanueva, A.; Esteller, M. \& Juarranz, A. Loss of E-cadherin mediated cell-cell adhesion as an early trigger of apoptosis induced by photodynamic treatment. J. Cell Physiol., 205(1):86-96, 2005.

Gooding, J. M.; Yap, K. L. \& Ikura, M. The cadherincatenin complex as a focal point of cell adhesion and signalling: new insights from three-dimensional structures. Bioessays, 26(5):497-511, 2004.

Gorouhi, F.; Davari, P. \& Fazel, N. Cutaneous and mucosal lichen planus: a comprehensive review of clinical subtypes, risk factors, diagnosis, and prognosis. Sci. World J., 2014:742826, 2014.
Hadzi-Mihailovic, M.; Raybaud, H.; Monteil, R. \& Jankovic, L. Expression of Fas/FasL in patients with oral lichen planus. J. BUON, 14(3):487-93, 2009.

Inki, P. \& Jalkanen, M. The role of syndecan-1 in malignancies. Ann. Med., 28(1):63-7, 1996.

Inki, P.; Larjava, H.; Haapasalmi, K.; Miettinen, H. M.; Grenman, R. \& Jalkanen, M. Expression of syndecan-1 is induced by differentiation and suppressed by malignant transformation of human keratinocytes. Eur. J. Cell Biol., 63(1):43-51, 1994.

Juuti, A.; Nordling, S.; Lundin, J.; Louhimo, J. \& Haglund, C. Syndecan-1 expression--a novel prognostic marker in pancreatic cancer. Oncology, 68(2-3):97-106, 2005.

Lei, L.; Tan, W. X.; Zhou, X. L. \& Zheng, P. E. Expression of Fas and Fas ligand in infiltrating lymphocytes in patients with oral lichen planus. Zhonghua Kou Qiang Yi Xue Za Zhi, 45(4):219-22, 2010.

Matsumoto, A.; Ono, M.; Fujimoto, Y.; Gallo, R. L.; Bernfield, M. \& Kohgo, Y. Reduced expression of syndecan-1 in human hepatocellular carcinoma with high metastatic potential. Int. J. Cancer, 74(5):482-91, 1997.

Mohtasham, N.; Anvari, K.; Memar, B.; Saghravanian, N.; Ghazi, N.; Bagherpour, A. \& Ramtin, M. Expression of Ecadherin and matrix metalloproteinase- 9 in oral squamous cell carcinoma and histologically negative surgical margins and association with clinicopathological parameters. Rom. J. Morphol. Embryol., 55(1):117-21, 2014.

Neppelberg, E.; Loro, L. L.; Oijordsbakken, G. \& Johamessen, C. Altered CD40 and E-cadherin expression--putative role in oral lichen planus. J. Oral Pathol. Med., 36(3):153-60, 2007.

Presland, R. B. \& Jurevic, R. J. Making sense of the epithelial barrier: what molecular biology and genetics tell us about the functions of oral mucosal and epidermal tissues. J. Dent. Educ., 66(4):564-74, 2002.

Reiland, J.; Sanderson, R. D.; Waguespack, M.; Barker, S. A.; Long, R.; Carson, D. D. \& Marchetti, D. Heparanase degrades syndecan-1 and perlecan heparan sulfate: functional implications for tumor cell invasion. J. Biol. Chem., 279(9):8047-55, 2004.

Santoro, A.; Majorana, A.; Bardellini, E.; Gentili, F.; Festa, S.; Sapelli, P. \& Facchetti, F. Cytotoxic molecule expression and epithelial cell apoptosis in oral and cutaneous lichen planus. Am. J. Clin. Pathol., 121(5):758-64, 2004. 
CARREON-BURCIAGA, R. G.; MENDOZA-ROAF, P.; AMEZCUA-ROSAS, G.; BOLOGNA-MOLINA, R.; GONZÉLEZ-MONTEMAYOR, T.; GONZÁLEZ-GONZÁLEZ, R. \& LEMÚSROJERO, O. Expresión de E-Cadherina y Syndecan-1 en liquen plano de piel y de mucosa oral. Int. J. Odontostomat., 8(3):439-445, 2014.

Saunders, S.; Jalkanen, M.; O'Farrell, S. \& Bernfield, M. Molecular cloning of syndecan, an integral membrane proteoglycan. J. Cell Biol., 108(4):1547-56, 1989.

Soukka, T.; Pohjola, J.; Inki, P. \& Happonen, R. P. Reduction of syndecan-1 expression is associated with dysplastic oral epithelium. J. Oral Pathol. Med., 29(7):308-13, 2000.

Stanley, M. J.; Liebersbach, B. F.; Liu, W.; Anhalt, D. J. \& Sanderson, R. D. Heparan sulfate-mediated cell aggregation. Syndecans- 1 and -4 mediate intercellular adhesion following their transfection into human $B$ lymphoid cells. J. Biol. Chem., 270(10):5077-83, 1995.

Sugerman, P. B.; Satterwhite, K. \& Bigby, M. Autocytotoxic T-cell clones in lichen planus. Br. J. Dermatol., 142(3):449-56, 2000.

Tsanou, E.; loachim, E.; Briasoulis, E.; Charchanti, A.; Damala, K.; Karavasilis, V.; Pavlidis, N. \& Agnantis, N. J. Clinicopathological study of the expression of syndecan1 in invasive breast carcinomas. Correlation with extracelular matrix components. J. Exp. Clin. Cancer Res., 23(4):641-50, 2004.

Zyada, M. M. \& Fikry, H. E. Immunohistochemical study of syndecan-1 down-regulation and the expression of P35 protein in oral lichen planus: a clinicopathologic correlation with hepatitis $C$ infection in the Egyptian population. Ann. Diagn. Pathol., 14(3):153-61, 2010.
Dirección para Correspondencia:

M. en C. Ramón Gil Carreón

Facultad de Odontología

Universidad Juárez del Estado de Durango-

Predio Canoas SN, Col.

Los Angeles, CP 34000

Durango

MÉXICO

Email: investigacion_odontologia_ujed@hotmail.com

Recibido : 26-09-2014

Aceptado: 23-10-2014 